\title{
Shear thickening of dense suspensions due to energy dissipation in lubrication layers between particles
}

\author{
L. Yu. Iskakova and A. Yu. Zubarev \\ Urals Federal University, Lenina Avenue 51, 620083, Ekaterinburg, Russia \\ (Received 24 May 2013; revised manuscript received 29 July 2013; published 13 September 2013)
}

\begin{abstract}
This paper deals with a theoretical study of the shear thickening effects in concentrated suspensions of non-Brownian particles. Our analysis shows that an increase of the shear rate of the suspension flow leads to a decrease of the mean thickness of the gaps between the nearest particles in dense suspensions. In turn, this leads to the growth of energy dissipation in these gaps, which means an increase of the suspension effective viscosity with the shear rate.
\end{abstract}

DOI: 10.1103/PhysRevE.88.032303

PACS number(s): $83.80 . \mathrm{Hj}$

\section{INTRODUCTION}

The classical Einstein theory [1] of suspension effective viscosity ignores any interactions between the particles of these systems and, therefore, deals with very dilute system suspensions, where the volume concentration of the particles does not exceed several percent. Generalization of the Einstein's theory, taking into account hydrodynamic and steric interactions of the particles, has been made in the works of Batchelor and Felderhof [2-5]. These theories lead to good agreement with experiments when the volume concentration of the particles is about $10 \%-15 \%$. One of the important qualitative conclusions of the theories [2-5] is that the suspension effective viscosity decreases with the shear rate $\dot{\gamma}$ of the suspension flow. This conclusion is supported by many experiments with moderately concentrated suspensions.

Experiments show that the rheophysics of dense suspensions (where the particles' volume concentration is $\gtrsim 40 \%$ $45 \%$ ) qualitatively differs from that of the moderately concentrated systems, described by the Batchelor and Felderhof theories. In part, the viscosity of the dense suspensions, at small shear rates, decreases with $\dot{\gamma}$, as in the moderately concentrated systems. However, starting with some critical magnitude of $\dot{\gamma}$, the viscosity increases with the shear rate. Growth of a fluid viscosity with the shear rate is known as the shear thickening effect.

For suspensions with a relatively small concentration of particles (about $40 \%-45 \%$ ), as a rule, quite smooth increase of the viscosity with $\dot{\gamma}$ is detected. In contrast, for suspensions with higher concentrations $(50 \%-60 \%)$ a sharp stepwise increase of the viscosity with $\dot{\gamma}$ is quite typical. For the systems with irregularly shaped particles the stepwise jump of the stress is significantly more than that for the suspensions of the smooth spheres [6]. Overviews of works on the rheology of the highly concentrated suspensions can be found in Refs. [7-13]. A typical sketch of the dependence of viscosity of concentrated suspensions on shear rate is shown in Fig. 1 (see also Ref. [8]).

The physical nature of the shear thickening effects has not been understood yet. Some attempts of their explanation are presented, for example, in Refs. [14-20]. Computer simulations [14] demonstrate the appearance of the chainlike structures whose axes are tilted about $45^{\circ}$ from the suspension velocity. These clusters appear due to the hydrodynamic forces which push the particles to each other. Under the action of the shear flow, these clusters rotate and disaggregate; simultaneously, new clusters appear, and so on. This hydrodynamic clusterization of the particles can induce the shear thickening phenomena in suspensions [14].

Shear thickening as a result of colloidal particles' clusterization, due to squeezing out of the particles (molecules) of the solvent from the interstice between the nearest particles, has been studied in computer simulations [21]. It was shown that the squeezing effect can lead to significant increase of the viscosity with the shear rate.

In [15] the rheological effects in dense suspensions have been explained by formation and destruction of some internal heterogeneous structures. Neither the type nor shape of these structures has been discussed in these works.

Another explanation of the shear thickening effects has been done in Refs. [16,17]. These experiments have shown that increase of the shear rate of the suspension flow first leads to appearance of the well-ordered layers of the particles. These layers are parallel to the flow velocity and perpendicular to the gradient of the flow. The further increase of the shear rate induces destruction of these layers and chaotization of the particles' space distribution. The shear thickening effect has been explained by the layers' chaotization. No estimates of dependence of the system viscosity on the shear rate have been done in $[16,17]$.

In the series of works [18-20] the rheophysics of the dense suspensions has been described on the basis of the dynamical theory of glasses. It was supposed that the suspension, under the action of the flow, is separated into regions with different concentration of the particles. Each of these regions has an effective temperature, which has nothing in common with the usual thermodynamic temperature of the system, but describes fluctuations of the particle position due to the random microscopical flows. This effective temperature depends either on the global shear rate or on the applied shear stress. The mathematical form of this dependence has been postulated. The approach of [18-20] allows reproducing the experimentally observed $\mathrm{N}$ - and S-like dependences of the macroscopical stress on the shear rate. However, it is based on many postulated assumptions and usage of many fit parameters.

One needs to admit that a general, universally recognized theory of the shear thickening effects in dense suspensions has not been developed yet. It is quite possible that, at least, several microscopical mechanisms can lead to this macroscopical 


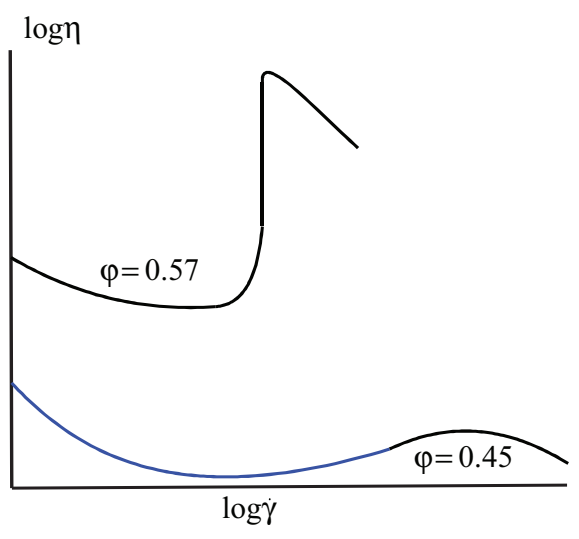

FIG. 1. (Color online) Schematic representation of concentrated suspension viscosity $\eta$ vs shear rate $\dot{\gamma}$.

phenomenon. Separate consideration and analysis of these mechanisms can allow us to clarify the microscopical physical nature of the shear thickening effects and the conditions when each of these mechanisms can dominate.

The aim of the presented work is theoretical analysis of the effect of the energy dissipation in thin lubrication layers between the closely situated particles on the macroscopical viscosity of the dense suspensions. Analysis shows that this mechanism can induce an increase of the viscosity with the shear rate of the suspension flow.

\section{PHYSICAL MODEL}

The classical theory of the effective viscosity of highly concentrated suspensions has been developed by Frankel and Acrivos [22]. According to this theory the effective viscosity $\eta$ of a dense suspension can be estimated as

$$
\eta=\eta_{0} C \frac{a}{\Delta} .
$$

Here $\eta_{0}$ is the viscosity of the carrier liquid, $a$ is the particle radius, $\Delta$ is the thickness of the liquid layer between the particles, and $C$ is a parameter, determined by mutual disposition of the particles. In Ref. [22] the estimate $C=9 / 8$ is recommended. Formula (1) is obtained under the assumptions that the particle concentration is high and the strong inequality $\Delta \ll a$ is held.

In the framework of the theory [22] the thickness $\Delta$ of the liquid layers between the particles is constant and determined by the stationary mutual disposition of the particles. However, in the shear flowing suspension the distance $\Delta$ between the nearest particles depends on time-the particles approach each other, move away, and so on. Therefore, dissipation of energy in the interparticle gaps, reflected in Eq. (1), also increases and then decreases with time. Thus, the correct formula (1) must include a mean magnitude of the ratio $a / \Delta$. Generally speaking, this means the ratio can depend on the macroscopical shear rate $\dot{\gamma}$.

While considering the mutual movement of the particles, illustrated in Fig. 2, two types of forces acting on them must be taken into account. The first one is the hydrodynamic Stokes force; the second one is the forces of potential repulsion between the particles. The last force prevents the irreversible

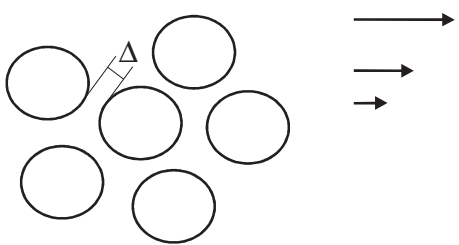

FIG. 2. Sketch of the suspension under consideration. Arrows illustrate the suspension flow.

aggregation of the particles under the action of the interparticle disperse forces.

When the particles approach each other, the Stokes and potential forces act in the opposite directions and the minimal distance $\Delta_{\text {min }}$ between the particles is determined by the competition between these forces. Obviously, the minimal distance $\Delta_{\text {min }}$ decreases with $\dot{\gamma}$. Therefore one can expect increase of the mean magnitude of the ratio $a / \Delta$, and thus growth of the effective viscosity $\eta$ with $\dot{\gamma}$.

In the next part of the work the effective viscosity $\eta$ is estimated on the basis of this physical consideration. For maximal simplification of calculations, we assume that the particles are identical non-Brownian hard spheres, their concentration is high enough, and the strong inequality $\Delta \ll a$ is held.

\section{MATHEMATICAL MODEL}

As in [22] we will focus on effects inside the thin liquid layer between two nearest particles. It is convenient to introduce a coordinate system with the origin in the center of one of them (say, of the first one), axis $O x$, aligned along the velocity of the macroscopical flow, and axis $O z$ directed along the gradient of this velocity. We will denote the radius vector directed to the center of the second particle as $\boldsymbol{r}$, and the angle between $\boldsymbol{r}$ and the axis $O x$ as $\theta$ (Fig. 3).

In the chosen coordinate system the components of the macroscopical flow velocity $\mathbf{v}$ are

$$
\mathrm{v}_{x}=\dot{\gamma} \cdot z, \quad \mathrm{v}_{z}=\mathrm{v}_{y}=0, \quad \dot{\gamma}=\text { const },
$$

where $\dot{\gamma}$ is the macroscopical shear rate of the flow. For convenience we will introduce the tensor $\boldsymbol{\Gamma}$ of the gradient of the macroscopical flow and present it as follows:

$$
\boldsymbol{\Gamma}=\mathbf{E}+\mathbf{\Omega} .
$$

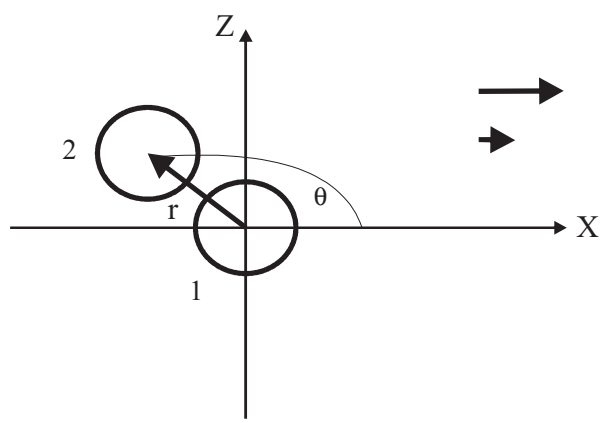

FIG. 3. Illustration of the interacting particles and the coordinate system. The horizontal arrows mean the same as in Fig. 1. 
Here $\mathbf{E}$ and $\boldsymbol{\Omega}$ are symmetrical and antisymmetrical parts of the tensor $\boldsymbol{\Gamma}$, respectively.

In the Cartesian coordinate system,

$$
\begin{aligned}
& \Gamma_{i j}=\frac{\partial \mathrm{v}_{i}}{\partial x_{j}}, \quad E_{i j}=\frac{1}{2}\left(\frac{\partial \mathrm{v}_{i}}{\partial x_{j}}+\frac{\partial \mathrm{v}_{j}}{\partial x_{i}}\right), \\
& \Omega_{i j}=\frac{1}{2}\left(\frac{\partial \mathrm{v}_{i}}{\partial x_{j}}-\frac{\partial \mathrm{v}_{j}}{\partial x_{i}}\right) .
\end{aligned}
$$

For the considered shear flow,

$$
\begin{aligned}
& \Gamma_{x z}=\dot{\gamma}, \quad \text { otherwise } \quad \Gamma_{i j}=0, \\
& E_{x z}=E_{z x}=\frac{1}{2} \dot{\gamma}, \quad \Omega_{x z}=-\Omega_{z x}=\frac{1}{2} \dot{\gamma} .
\end{aligned}
$$

In order to consider the relative motion of the particles we will use the Batchelor-Green theory [23] of the hydrodynamical interaction of two particles in a suspension of hard spheres. This theory takes into account only pair interactions between the particles, ignoring the effect of the third and other particles on the relative motion of each pair. That is why for concentrated suspensions this model can be considered only as a first approximation, which allows us to estimate the dissipation effects in the thin liquid layer between two particles.

According to the theory [23], the relative motion of the particles consists of the following components: (i) convective motion due to the macroscopical flow of suspension, (ii) the motion under the action of potential force of the particles' interaction, and (iii) the diffusion motion.

The relative velocity of the convective motion can be presented as [23]

$$
\mathbf{V}=\mathbf{r} \cdot \mathbf{\Gamma}-\left\{A \frac{\mathbf{r} \cdot \mathbf{r}}{r^{2}}+B\left(\mathbf{I}-\frac{\mathbf{r} \cdot \mathbf{r}}{r^{2}}\right)\right\}(\mathbf{r} \cdot \mathbf{E}),
$$

where $A$ and $B$ are coefficients, determined below; $\mathbf{I}$ is the unit tensor defined as

$$
I_{i, j}=\left\{\begin{array}{ll}
1, & i=j \\
0, & i \neq j
\end{array} .\right.
$$

The sign $\cdot$ means the dyadic multiplication, defined according to the following rule:

For two vectors $\mathbf{a} \cdot \mathbf{b}=a_{i} b_{j}$;

For a vector and a tensor $\boldsymbol{\beta} \cdot \mathbf{a}=\beta_{i j} a_{j}$.

The Einstein rule of summation over the coordinate indices is used here.

Neglecting the diffusion motion, one can write an equation of the relative motion of the particles in the following form [23]:

$$
\frac{d \mathbf{r}}{d t}=\mathbf{V}-\boldsymbol{\beta}(\mathbf{r}) \cdot \nabla U
$$

Here $\beta$ is the coefficient of the relative mobility of the particles; $U$ is the potential of their interaction. According to the theory $[23,24]$ the coefficient $\beta$ can be presented as

$$
\begin{aligned}
\boldsymbol{\beta}(\mathbf{r}) & =2 \beta_{0}\left[G(r) \frac{\mathbf{r} \cdot \mathbf{r}}{r^{2}}+H(r)\left(\mathbf{I}-\frac{\mathbf{r} \cdot \mathbf{r}}{r^{2}}\right)\right], \\
\beta_{0} & =\frac{1}{6 \pi \eta_{0} a} .
\end{aligned}
$$

The Einstein rule of summation over the repeating indices is used here.
In the asymptotic $r \rightarrow 2 a$ the functions $A, B, G$, and $H$ can be estimated as [23,24]

$$
\begin{aligned}
& G \approx 4(\rho-1), \quad H \approx 0.4, \\
& A \approx 1-8.15(\rho-1), \quad B \approx 0.406+\frac{0.78}{\ln 2(\rho-1)},
\end{aligned}
$$

where $\rho=r / 2 a$.

Let us introduce a spherical coordinate system with the radius $r$ and polar angle $\theta$ (Fig. 2). Assuming that the potential interaction between the particles is central, i.e., the potential $U$ depends only on the distance $r$ between the particle centers, Eq. (2) can be rewritten in the following form:

$$
\begin{aligned}
\frac{d \rho}{d t} & =w_{r}-\frac{1}{2 a} \beta_{r r} \frac{\partial U}{\partial r}, \quad \beta_{r r}=2 \beta_{0} G(r), \\
\rho \frac{d \theta}{d t} & =w_{\theta}, \quad \rho \sin (\psi) \frac{d \psi}{d t}=w_{\psi},
\end{aligned}
$$

where

$$
\begin{aligned}
w_{r} & =8.15 \dot{\gamma} \rho(\rho-1) \sin \theta \cos \theta \cos \psi, \\
w_{\theta} & =-\dot{\gamma} \rho\left[\sin ^{2} \theta+\frac{B}{2}\left(\cos ^{2} \theta-\sin ^{2} \theta\right)\right] \cos \psi, \\
w_{\psi} & =\dot{\gamma} \rho \frac{B}{2} \cos \theta \sin \psi, \\
\mathbf{w} & =\frac{\mathbf{V}}{2 a} .
\end{aligned}
$$

It should be stressed that the formulas (5) have been obtained in the asymptote $\rho \rightarrow 1$.

In concentrated suspensions the approximation of the pair interaction between the particles, even in the asymptotic of their close disposition, can lead to qualitatively incorrect results. Indeed, in the case of the weak shear flow, when the force of the potential repulsion $-\nabla U$ dominates the hydrodynamic force, Eqs. (3)-(5) lead to infinite growth of the interparticle distance $\rho$ with time. Obviously, in the dense systems too large an increase of the distance between the nearest particles is impossible because of the restricting effect of the other particles. Thus the multiparticle effects, which do not allow the particles to move far away under the action of the potential repulsion, must be taken into account here.

There is no way to solve this problem strictly. In order to get physically reasonable estimates, we will use here the concept of the model of a tube, suggested by de Gennes and developed by Doi and Edwards [25]. This model allows the taking into account of the effect of the steric interaction in the dense polymers and liquid-crystal systems on dynamical properties of these systems. In the framework of this model, relative motion of the particles (monomers, molecules) is restricted by a tube. The shape and size of this tube is determined by the concentration and features of the space disposition of the particles.

Unlike the de Gennes-Doi-Edwards theory, the Brownian motion of the particles does not play any role in the considered suspensions. In part, in the model [25] the monomers of a polymer chain, due to Brownian motion, can move in the direction of the macroscopical flow and in the opposite direction as well. That is why the tube is symmetrical with respect to the flow. In the situation under consideration, the motion of particle 2, illustrated in Fig. 2, in the direction 


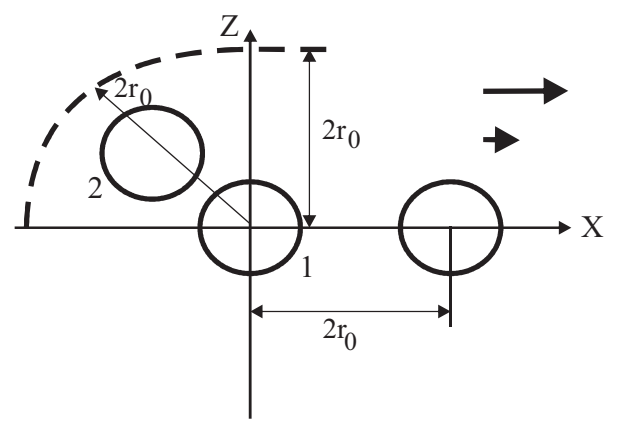

FIG. 4. Sketch of the tube of possible positions of particle 2 above particle 1 .

opposite to the flow velocity (i.e., from the right to the left), is impossible. At the same time the effect of the other particles significantly restricts the vertical (in Fig. 2) motion of particle 2. In contrast, its motion in the direction of the flow (toward the right in Fig. 2) is not restricted, since other particles, situated to the right of particle 1 , move in the same direction.

The size of the tube, where particle 2 can move without significant resistance from the other particles except particle 1 , is determined by the mean distance $r_{0}$ between the centers of the nearest particles. In the order of magnitude this distance can be estimated as (see, for example, [22])

$$
r_{0}=2 a\left(\frac{\varphi_{m}}{\varphi}\right)^{1 / 3}
$$

where $\varphi$ is the volume concentration of the particles in the suspension; $\varphi_{m}$ is the dense packing concentration. Depending on the type of the particle spatial disposition, $\varphi_{m}$ varies from 0.6 to 0.72 . Thus, the tube of the possible displacement of particle 2 near particle 1 must be restricted left of particle 1 by the semisphere whose center is in the center of particle 1 . The radius $r_{\mathrm{c}}$ of this semisphere can be estimated as $r_{c} \sim 2 r_{0}$. To the right of particle 1 , the displacement of particle 2 is restricted by the cylinder with the radius $r_{c}$ and the axis, aligned along the flow velocity. This tube is shown in Fig. 4.

We must take into account that when particle 2 approaches the tube boundary, the forces of potential repulsion from the other particles will act on particle 2 .

Taking this into account, we can modify the Eq. (4a) as follows:

Left of particle $1(\pi / 2<\theta<\pi)$,

$$
\frac{d \rho}{d t}=w_{r}-\frac{2}{a^{2}} \beta_{0}(1-\rho) \frac{\partial}{\partial \rho}\left[U(\rho)-U\left(\rho_{c}-\rho\right)\right]_{r} .
$$

Right of particle $1(0<\theta<\pi / 2)$,

$$
\begin{aligned}
\frac{d \rho}{d t}= & w_{r}-\frac{2}{a^{2}} \beta_{0}(1-\rho) \\
& \times\left\{\frac{\partial}{\partial \rho}\left[U(\rho)-\sin \theta \frac{\partial}{\partial z} U\left(\rho_{c}-z\right)\right]\right\}, \\
z= & \rho \sin \theta, \quad \rho_{c}=\frac{r_{c}}{2 a} .
\end{aligned}
$$

Numerical solution of the system (4b), (7) allows us to determine $\rho$ and $\theta$ as functions of time $t$. In turn, this allows us to determine the gap thickness $\Delta(\theta)=2 a[\rho(\theta)-1]$ as a function of the time $t$.
Now we must take into account that particle 2, moving in the direction right from particle 1 , in a certain time which depends on the velocity of the particle's relative motion, will approach particle 3 (Fig. 4).

Obviously, at the moment when particle 2 is to the right of particle 1 and the distance $r$ between them exceeds $r_{0}$, these particles cannot be considered as the nearest ones, since particle 2 will be closer to particle 3 .

In order to solve Eqs. (5) and (7), one needs to determine the initial conditions of these equations. It is reasonable to estimate the dimensionless initial distance $\rho(0)$ between particles 1 and 2 as the mean distance $\rho_{0}=r_{0} / 2 a$ between the particles in the suspension. As for the initial angles $\theta_{0}$ and $\psi_{0}$, one can suppose that they are equiprobably distributed in the intervals $\pi / 2<\theta_{0}<\pi, 0<\psi_{0}<\pi$. The chosen region for the azimuth angle $\psi_{0}$ corresponds to the upper part of Fig. 3, where particle 2 moves relatively to particle 1 from the left to the right. In the lower half plane, corresponding to the interval $\pi<\psi<2 \pi$, this motion is in the direction from the right to the left. It is obvious that the dissipation of the energy, which takes place due to the particle motion in the lower half plane of Fig. 3, is the same as the one in the upper half plane. That is why, for simplicity, we restrict ourselves by consideration of the energy dissipation in the upper half plane, corresponding to the interval $0<\psi_{0}<\pi$.

Thus, the physical (measured) magnitude of the effective viscosity $\eta$ must be determined by the magnitude of the inverse thickness $1 / \Delta$ of the interparticle gap, averaged over time from the onset of the motion to the time $T$, determined by the equality $\rho(T)=\rho_{0}$. Taking into account Eq. (1), we can write

$$
\begin{aligned}
\eta= & \eta_{0} C a \frac{1}{\pi} \int_{0}^{\pi} d \psi_{0} \int_{\pi / 2}^{\pi} \sin \left(\theta_{0}\right) d \theta_{0} \frac{1}{T\left(\theta_{0}, \psi_{0}\right)} \\
& \times \int_{0_{0}}^{T} \frac{1}{\Delta\left(t, \theta_{0}, \psi_{0}\right)} d t .
\end{aligned}
$$

To calculate the integral (8), first, we must solve numerically Eqs. (4b) and (7) and also determine the time $T$ from the equation $\rho(T)=\rho_{0}$. These equations can be solved numerically.

\section{RESULTS OF CALCULATIONS}

Let us suppose that the potential $U$ of the particle interaction is the electrostatic potential due to double electrical layers near the particle surface. We will use the following simple form of this potential [26,27]:

$$
U=-\frac{(Z q)^{2}}{8 \pi \varepsilon_{0} \varepsilon \kappa^{2} a^{3}} \ln \{1-\exp [-2 a(\rho-1) \kappa]\},
$$

which corresponds to the condition of the constant charge of the particles while changing the distance between them. Here $1 / \kappa$ is the Debye thickness of the double layer, $Z q$ is the charge of the particle surface, $q$ is the electron charge, $\varepsilon$ is the relative dielectric permeability of the carrier liquid, and $\varepsilon_{0}$ is the vacuum permeability. 


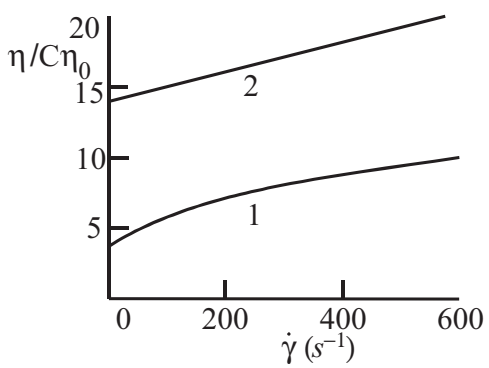

FIG. 5. Results of calculations of the effective viscosity $\eta$ vs the shear rate $\dot{\gamma}$. Parameters of the system: $a=0.5 \times 10^{-6} \mathrm{~m}, \kappa a=20$, $\varepsilon=70, \eta_{0}=10^{-3} \mathrm{Pas}, Z=2500$. Lines 1 and 2 correspond to $\varphi=$ $0.6 \varphi_{m}$ and $0.9 \varphi_{m}$, respectively.

The results of calculations of the effective viscosity $\eta$ vs the shear rate $\dot{\gamma}$ for two concentrations $\varphi$ of the particles are given in Figs. 5 and 6. The used parameters of the potential $U$ are quite typical for many aqueous suspensions [26,27].

The results demonstrate an increase of the effective viscosity with the shear rate. In other words, the mechanism of the energy dissipation in the thin lubrication layers between the particles provides a quite measurable shear thickening effect. The magnitude of this effect significantly depends on the Debye thickness of the double electrical layer near the particles. When the layers' thickness is small as compared with the particle diameter, the energy dissipation in the thin lubrication layers between the particles leads to an increase of the effective viscosity $\eta$ (in the considered region of $\dot{\gamma}$ ) about several times and even more than an order of magnitude. Qualitatively this conclusion is in agreement with the experimental results, presented in $[8,11-13,15]$. In part, experiment [11] demonstrates strong, i.e., more than an order of magnitude, growth of the suspension viscosity with an increase of the $p \mathrm{H}$ of the system. This means compression of the Debye layers and, therefore, increase of the complex $\kappa a$. Especially strong growth has been observed on the part of the rheograms, corresponding to the shear thickening behavior of the suspension. A significant increase, on the shear thickening part of the rheogram, of the viscosity of a concentrated suspension with the particle radius $a$, has been observed in experiments [12]. This conclusion is in agreement with the results, presented in Fig. 6. Experiments [13] show strong, more than an order of magnitude, increase of suspension

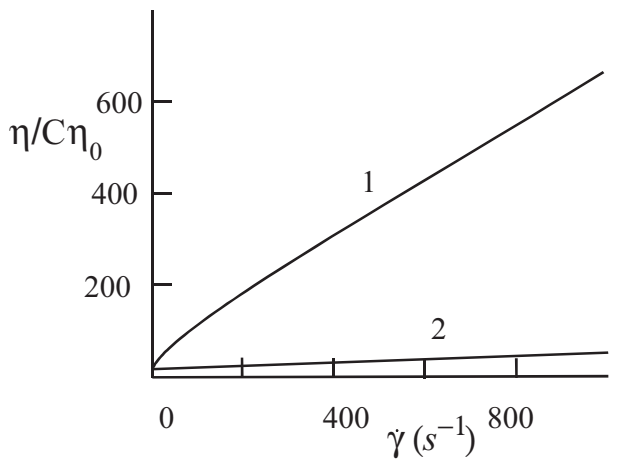

FIG. 6. Same as in Fig. 5 when $\varphi=0.7 \varphi_{m}$. Lines 1 and 2 correspond to $\kappa a=100$ and 20 , respectively. viscosity with the shear rate. Curve 1 in Fig. 6 indicates that this growth can take place due to the considered mechanism of the energy dissipation in the thin lubrication layers between the suspended particles.

Unfortunately it is impossible to compare the theoretical and experimental results quantitatively. The fact is, practically all experiments demonstrate significant decrease of the suspension viscosity with the shear rate (shear thinning effect) when the rate is small enough. The shear thickening behavior is observed only when the shear rate exceeds some critical magnitude. Therefore, in the real suspensions, the internal mechanisms which lead to the shear thickening effects compete with the ones which induce the shear thinning phenomena. All these mechanisms must be taken into account in order to compare quantitatively a theory with the experiments. However, a quantitative theory of the shear thinning mechanisms in concentrated suspensions has not been developed yet. One needs to note that experiments [13] show a decrease of the threshold (for the beginning of the shear thickening behavior) values of the shear rate while the suspension $p \mathrm{H}$ increases. This is in agreement with the results, presented in Fig. 6, which indicate that compression of the double electrical layers near the particles (i.e., increase of the parameter $\kappa$ ) leads to the more sharp growth of the viscosity $\eta$ with the shear rate $\dot{\gamma}$.

It should be noted that computer simulations [28] have demonstrated significant effect of combination of thermodynamic and lubrication forces between the close particles on the shear thickening phenomena in the dense suspensions. Results of our analysis are in principal agreement with these simulations.

\section{CONCLUSIONS}

We have considered a microscopic mechanism of the macroscopic shear thickening effect in the dense suspensions of non-Brownian spherical particles. This model is based on the idea of the theory [21] that the main dissipation of energy in these systems takes place in the thin liquid layers between the nearest particles. According to [21] the suspension effective viscosity is proportional to the ratio $a / \Delta$, where $a$ is the particle radius and $\Delta$ is the layer thickness. We take into account that, in the shear flowing suspensions, the relative motion of the particles leads to periodical decrease and increase of $\Delta$. Our analysis shows that the mean value of the ratio $1 / \Delta$ is an increasing function of the shear rate $\dot{\gamma}$. This means that the effect of the energy dissipation in the interparticle layers leads to an increase of the suspension effective viscosity with the shear rate.

In the experiments the described microscopical mechanism of the macroscopical shear thickening effect can act simultaneously with the other internal mechanisms, including those which lead to the shear thinning phenomena. For example, with formation and destruction of the ordered layers of the particles [8-10,15,16], with appearance of the chainlike structures [13], with the space separation of the suspension into regions with different concentration of the particles [17-19], and possibly with other mechanisms.

Since strict theoretical study of rheological effects in concentrated suspensions is impossible, goal-seeking 
experimental and computer investigations are necessary to find out when each of these microscopical mechanisms plays the main role in formation of the macroscopical rheological effect.

It should be stressed that the presented model deals only with the mechanism of the shear thickening effect and does not consider the mechanisms of the shear thinning phenomena (see Fig. 1). That is why this model cannot describe the transition from the thinning to the thickening effects. Therefore, it does not allow estimating the threshold (for these transitions) magnitudes of the shear rate. A theoretical description of the shear thinning phenomena and, therefore, estimation of the threshold magnitudes of the shear rate, requires a special study.

\section{ACKNOWLEDGMENTS}

This work has been done with the support of the Russian Fund of Fundamental Investigations, Grants No. 1201-00132, No. 13-02-91052, and No. 13-01-96047; by the Federal Goal Program of Russian Federation, Agreement No. 14.A18.21.0867; and the program of the Ministry of Education of Russian Federation, Project No. 2.1267.2011.
[1] A. Einstein, Ann. Phys. (Leipzig, Ger.) 17, 459 (1905).

[2] G. K. Batchelor and J. T. Green, J. Fluid Mech. 56, 401 (1972).

[3] G. K. Batchelor, J. Fluid Mech. 86, 97 (1977).

[4] U. Felderhof and R. Jones, Physica A 146, 417 (1987).

[5] B. Cichocki and U. Felderhof, J. Chem. Phys. 101, 7850 (1994).

[6] E. Brown and H. M. Jaeger, Phys. Rev. Lett. 103, 086001 (2009).

[7] A. J. C. Ladd, J. Chem. Phys. 93, 3484 (1990).

[8] H. A. Barnes, J. Rheol. 33, 329 (1989).

[9] W. J. Frith, P. d'Haene, R. Buscall, and J. Mewis, J. Rheol. 40, 531 (1996).

[10] J. S. Lee and N. J. Wagner, Rheol. Acta 42, 199 (2003).

[11] G. V. Franks, Z. Zhon, and N. J. Duin, J. Rheol. 44, 759 (2000).

[12] B. J. Maranzano and N. J. Wagner, J. Chem. Phys. 114, 10514 (2001).

[13] V. T. O. Brien and M. E. Mackay, J. Rheol. 46, 557 (2002).

[14] G. Bossis and J. F. Brady, J. Chem. Phys. 91, 1866 (1989).

[15] D. Quemada, Eur. Phys. J. Appl. Phys. 1, 119 (1998); 2, 175 (1998); 3, 301 (1998); 5, 191 (1998).

[16] R. Hoffman, J. Colloid Interface Sci. 46, 491 (1974).
[17] R. Hoffman, J. Rheol. 42, 111 (1998).

[18] M. Fuchs and M. E. Cates, Phys. Rev. Lett. 89, 248304 (2002).

[19] D. A. Head, A. Ajdari, and M. E. Cates, Phys. Rev. E 64, 061509 (2001).

[20] D. Head, A. Ajdari, and M. Cates, Europhys. Lett. 55, 120 (2002).

[21] J. Delhommelle and J. Petravic, J. Chem. Phys. 123, 074707 (2005)

[22] N. F. Frankel and A. Acrivos, Chem. Eng. Sci. 22, 847 (1967).

[23] G. K. Batchelor and J. T. Green, J. Fluid Mech. 56, 375 (1972).

[24] G. K. Batchelor, J. Fluid Mech. 74, 1 (1976).

[25] M. Doi and S. F. Edwad, The Theory of Polymer Dynamics (Clarendon Press, Oxford, 1979).

[26] D. Quemada and C. Berli, Adv. Colloid Interface Sci. 98, 51 (2002).

[27] W. B. Russel, Colloidal Dispersions (Cambridge University Press, Cambridge, 1989).

[28] J. R. Melrose and R. C. Ball, J. Rheol., 48, 937 (2004). 